\title{
Intrinsic localized modes: Discrete breathers. Existence and linear stability
}

\author{
J.L. Marín ${ }^{\mathrm{a}, *}$, S. Aubry b,1, L.M. Floría ${ }^{\mathrm{a}}$ \\ ${ }^{a}$ Departamento de Física de la Materia Condensada, Instituto de Ciencia de Materiales de Aragón, \\ Universidad de Zaragoza, 50009 Zaragoza, Spain \\ ${ }^{\mathrm{b}}$ Laboratoire Léon Brillouin (CEA-CNRS), CE Saclay, 91191 Gif-sur-Yvette Cedex, France
}

\begin{abstract}
We present some examples of detailed analysis of intrinsic localized modes in lattices, using the accurate numerical methods derived from the proof of existence of MacKay-Aubry. We report on some improvements on the methods, which are then used to the fullest to obtain the Floquet analysis of the breather solutions. Such calculations are possible taking into account the whole lattice, without any approximations. This yields an unprecedented detail of the mechanisms that govern instabilities in discrete breathers, giving a complete picture of the interplay between localized ("internal") and extended ("phonon-like") instabilities. Copyright (C) 1998 Elsevier Science B.V.
\end{abstract}

Keywords: Breathers; Intrinsic localization; Floquet analysis; Extended dynamical systems

\section{Introduction}

Intrinsic localized modes (ILMs), otherwise known as discrete breathers, are a relatively recent discovery. Although there had been many studies on localized modes on nonlinear models (where the closest in relation to ILMs could be the ones on the discrete self-trapping equation [1]), it was only in the works of Takeno and coworkers [2-4] that the phenomenon was realized to be of great genericity in lattices. They obtained analytic approximations to periodic, highly localized vibrations in several nonlinear lattice models, many of which proved to be long-lived in numerical simulations. Later, moving localized pulses which also maintained their localization for very long times were discovered [5,6].

Both nonlinearity and the discreteness were identified as key ingredients for the existence of ILMs. The term "intrinsic localization" was adequately coined to describe this new phenomenon, which allows localized vibration in perfectly homogeneous lattices, without disorder or defects. On the other hand, the similarity between ILMs and their solitonic analogs in continuum models has brought about the term "discrete breathers", or simply breathers.

\footnotetext{
* Corresponding author. E-mail: marin@wanda.unizar.es.

${ }^{1}$ E-mail: aubry@bali.saclay.cea.fr.
} 
Of course, these solutions do not belong in general to integrable systems, and their properties seem to be far from solitonic. But considering the extremely high genericity of this phenomenon (in contrast to the highly improbable integrable systems), it is not too adventurous to say that discrete breathers will shortly attract more study and attention than pure "bonafide" breathers.

The most rigorous results on ILMs are those of MacKay-Aubry [7], where the stationary breathers are proved to exist in roughly any nonlinear lattice, provided only some non-resonance conditions and some upper limits on the coupling strength (or equivalently, the discreteness). The enormous power of the result lies in that it proves the genericity of ILMs, giving sufficient conditions for their existence, and even predicting the linear stability for some of them. The proof involves concepts of anti-integrability which were previously applied to other different physical systems (for a review, see [8,9]). Here, it deals with lattices at the anti-integrable or anti-continuum limit, where the oscillators are uncoupled. Thus the proof applies directly to lattices with a nonlinear on-site potential (subsequently called Klein-Gordon, or KG lattices), and only indirectly to lattices without on-site terms (Fermi-Pasta-Ulam, or FPU lattices). Extensions of the existence theorems for these cases are currently being worked on. On the other hand, the oscillators considered in this proof can be rotators (see the rotobreathers in [10,11]) and even forced and damped models (see $[11,12]$ ), systems in which ILMs were not previously described.

It should not be surprising that the calculation of discrete breathers has to be numerical. Considering the freedom in the choice of model potentials, it is doubtful that we could ever obtain closed analytical forms for these solutions. Beyond the initial (approximate) technique of lattice Green functions [4], a powerful numerical procedure to obtain accurate solutions has been developed in [11], following closely the principles of anti-integrability. Another highaccuracy method has been described in [13], but it lacks the certainty offered by continuation from well-known anti-continuum limits.

In the present paper we want first to report on a significant addition to the numerical techniques to obtain breathers as described in [11]. Then we present the first accurate studies of the linear stability of elementary breathers in several models, describing the numerical technique in detail. We describe all the possible scenarios for instabilities in some of the simplest solutions, the one-site and two-site breathers.

\section{Numerical methods for calculating breathers}

The numerical methods described in [11] to obtain localized modes consist basically in a continuation from the trivial solutions that we have in the anti-continuum limit of the lattice. We start with a KG lattice with an on-site anharmonic potential $V$ and a coupling $W$ (harmonic or not) parametrized with a discreteness parameter $\lambda$, with Hamiltonian:

$$
H=\sum_{i} \frac{1}{2} \dot{u}_{i}^{2}+V\left(u_{i}\right)+\lambda W\left(u_{i}, u_{i+1}\right) .
$$

The theorems in [7] prove the existence of periodic, localized solutions (breathers) in systems like (2.1) as continuation (in $\lambda$ ) from periodic orbits at $\lambda=0$ (the anti-continuum limit of the lattice). For FPU lattices one can also use this result in numerical calculations of breathers, as explained in [11], but the existence is in principle not assured.

The continuation procedure is effectively accomplished by a Newton-Raphson root finding method, applied to the suitable system. Ref. [11] gives two possibilities: to work on the Fourier-transformed space, or to work with some adequate restriction of the Poincaré map of the periodic orbit in the lattice. The Fourier technique has the difficulty of calculating the nonlinear terms, and therefore it was applied only to potentials with low power nonlinearities, where simple "convolutions" of the coefficients were feasible to calculate. The possibility of using the fast Fourier transform (FFT) was mentioned (as in [13]), but was not actually tried. Here we report on the successful use of the 
FFT in the framework of the Newton methods to obtain breathers. The addition of one more method to calculate breathers would be important in itself if just to double-check other methods, but this one happens to be both accurate and efficient, and thus could rival the use of the Poincaré map method.

The procedure goes as follows. For simplicity in the notation we will consider a 1D lattice, but the method works in any dimension. The equations of motion read

$$
\ddot{u}_{i}(t)+V^{\prime}\left(u_{i}(t)\right)+\lambda\left(W^{\prime}\left(u_{i-1}(t), u_{i}(t)\right)+W^{\prime}\left(u_{i}(t), u_{i+1}(t)\right)\right)=0,
$$

and we seek a spatially localized and periodic solution of this system, with a frequency $\omega_{\mathrm{b}}$. Therefore we can write the solution as a Fourier series:

$$
u_{i}(t)=\sum_{k} x_{k}^{(i)} \mathrm{e}^{-\mathrm{i} k \omega_{\mathrm{b}} t}, \quad \text { with } x_{-k}^{(i) *}=x_{k}^{(i)},
$$

where the asterisk denotes complex conjugation. The original equations then become

$$
-k^{2} \omega_{\mathrm{b}}^{2} x_{k}^{(i)}+V_{k}^{\prime(i)}+\lambda\left({W_{k}^{\prime}}^{(i-1, i)}+W_{k}^{\prime(i, i+1)}\right)=0,
$$

where $V_{k}^{\prime}$ and $W_{k}^{\prime}$ have the obvious meaning. Of course, in the calculations we will use a cut-off value $k_{\max }$ in the harmonic series that will be determined by our precision requirements and the particular behavior of the nonlinear potentials.

Following the existence theorem, we consider the possible configurations of sites at $\lambda=0$ that can be continued into localized solutions. For the Hamiltonian ${ }^{2}$ case we are considering here, the continuation is assured for configurations in which each site is either at rest, or oscillating with frequency $\omega_{\mathrm{b}}$. The mutual phase differences for those which oscillate can be only 0 or $\pi$ (which in our case means that we can work with real coefficients). Then (2.4) form the set of algebraic equations in the coefficients $x_{k}^{(i)}$ that we can solve with a Newton-Raphson method, following the breather solution in $\lambda$. The difficulty now lies in evaluating the terms $V_{k}^{\prime}, W_{k}^{\prime}$ from the coefficients $\left\{x_{k}^{(i)}\right\}$. Here is where we use the FFT: for each site (i), we construct the time-dependent function $u(t)$ as in Eq. (2.3). To make an optimal use of the FFT, we should "sample" $u(t)$ at $2 k_{\max }$ equidistant values of time, from $t=0$ to $t=2 \pi / \omega_{\mathrm{b}}$ (inverse FFT). From these samples of $u(t)$ we readily compute samples of $V^{\prime}\left(u_{i}(t)\right)$ and $W^{\prime}\left(u_{i}(t), u_{i+1}(t)\right)$. We feed these to the FFT to finally obtain the coefficients $V_{k}^{\prime}$ and $W_{k}^{\prime}$.

It should be noted that with this sampling the FFT is very accurate in producing the Fourier coefficients needed. In particular, it would return the coefficients of $u(t)$ almost exactly. The limitation lies in that the smaller coefficients obtained from an FFT are only accurate to machine precision relative to the magnitude of the biggest one. However this operation is performed locally at each site (or neighboring sites), and therefore that problem does not affect much the quality of the breather solution as a whole. On the other hand, this "feature" of the FFT will allow us to choose the maximum $k_{\max }$ which is useful in the calculation: it suffices to probe the single oscillator for the decay of the series $\left\{V_{k}\right\}$ (or $\left\{W_{k}\right\}$ ) with $k$ for a given frequency $\omega_{\mathrm{b}}$, to get some estimates.

We mention finally that this method has been successfully implemented and its quality and efficiency have been assessed. Its applicability is just as wide as the Poincaré map method [11], and can actually compete with it. ${ }^{3}$ All subsequent calculations presented in this paper have been obtained with the method presented here.

\footnotetext{
${ }^{2}$ For simplicity in the exposition, we consider only the time-reversible Hamiltonian case, leaving out, for instance, rotobreathers. See [11] for the details.

${ }^{3}$ Note that it is also applicable for rotating modes (rotobreathers), due to their step-periodic character.
} 


\section{Stability analysis of stationary breathers}

One of the aspects about intrinsic localized modes that was not clarified up to now is the linear stability. Earlier studies had to rely either on numerical simulation [14] or on excessive approximations [15]. With the new highaccuracy methods available, one can afford to perform the full analysis. This is just the standard Floquet analysis of the periodic solution, as found in many textbooks (see for example [16]). With knowledge of the breather solution $\left\{u_{i}(t)\right\}$, one integrates the linearized equations of motion corresponding to the original system (2.2):

$$
\ddot{\epsilon}_{i}+V^{\prime \prime}\left(u_{i}(t)\right) \epsilon_{i}+\lambda\left[W^{\prime \prime}\left(u_{i-1}(t), u_{i}(t)\right)\left(\epsilon_{i}-\epsilon_{i-1}\right)+W^{\prime \prime}\left(u_{i}(t), u_{i+1}(t)\right)\left(\epsilon_{i}-\epsilon_{i+1}\right)\right]=0,
$$

where, for simplicity, we have assumed that $W\left(u, u^{\prime}\right)$ has a dependency of the type $W\left(u-u^{\prime}\right)$. Other cases would work analogously.

In a lattice calculation with $N_{\max }$ sites, we have to integrate Eqs. (3.1) over a period $T_{\mathrm{b}}=2 \pi / \omega_{\mathrm{b}}$, for each of the $2 N_{\max }$ initial conditions of a vector basis of the $2 N_{\max }$-dimensional vector space formed by the variables $\left\{\epsilon_{i}, \dot{\epsilon}_{i}\right\}$. With this calculation we obtain the monodromy matrix $\mathcal{M}$ of the periodic breather,

$$
\left(\begin{array}{c}
\epsilon_{i} \\
\dot{\epsilon}_{i}
\end{array}\right)_{t=T_{\mathrm{b}}}=\mathcal{M}\left(\begin{array}{c}
\epsilon_{i} \\
\dot{\epsilon}_{i}
\end{array}\right)_{t=0},
$$

whose eigenvalues are the Floquet multipliers. They contain all the information about the linear stability of the periodic solution. In general, linear stability is assured when all the eigenvalues lie inside the unit circle. But for Hamiltonian systems it is not so simple: they lie right on the unit circle when the solution is stable, and when it is unstable, quadruplets of eigenvalues appear out of the unit circle. This is so because for each eigenvalue $v$ there has to be present also $v, v * 1 / v, 1 / v *$, since the system is real and Hamiltonian (symplectic). If $v$ is on the real axis then we only have $v$ and $1 / v$ out of the circle.

Since we will be analyzing the stability of one-parameter $(\lambda)$ families of breather solutions, we will observe a continuous movement of the corresponding eigenvalues on the complex plane. Then it is evident that if we start from a stable solution, we can only reach an instability if we observe collisions of eigenvalues on the unit circle. ${ }^{4}$ This problem is therefore analogous to that of the stability of parametric oscillations. Krein theory complements the picture for the Hamiltonian case, pointing out which collisions of eigenvalues are "forbidden" to cause instability. For the details on the theory, see $[17,18]$.

Analyzing the breather solutions in terms of the coupling parameter also shows the benefits in clarity of the antiintegrable approach. It starts analyzing the solutions from a well-known limit, so that any subsequent bifurcations, merges, etc. are easily spotted. At $\lambda=0$ each oscillating site will contribute two eigenmodes at +1 , while each site at rest will contribute two eigenmodes $\mathrm{e}^{ \pm \mathrm{i} \phi_{0}}$ at an angle $\phi_{0}=2 \pi \omega_{0} / \omega_{\mathrm{b}}$. From now on, we assume the potentials $V$ and $W$ normalized such that the rest frequencies are $\omega_{0}=1$. Assume for simplicity some anti-integrable configuration intended for a localized breather, i.e., all sites at rest except for a very few of them which are close to each other. When the coupling is switched on, most of the eigenvalues departing from $\phi_{0}$ (the majority) will span values $\mathrm{e}^{ \pm \mathrm{i} \phi}$ very close to those given by the linear phonon band of the lattice,

$$
\omega_{q}^{2}=1+4 \lambda \sin ^{2}(q / 2), \quad \phi_{q}=2 \pi \frac{\omega_{q}}{\omega_{\mathrm{b}}}
$$

while the rest are eigenmodes with localized eigenvector, whose behavior is unpredictable and has to be numerically computed. All those coming from +1 are obviously localized, and some others coming from $\phi_{0}$ may become progressively more localized with increasing $\lambda$ (see [24]).

\footnotetext{
${ }^{4}$ A remarkable consequence of this, as pointed out in [7], is that all one-site breathers have a non-vanishing window of stability with origin in $\lambda=0$.
} 

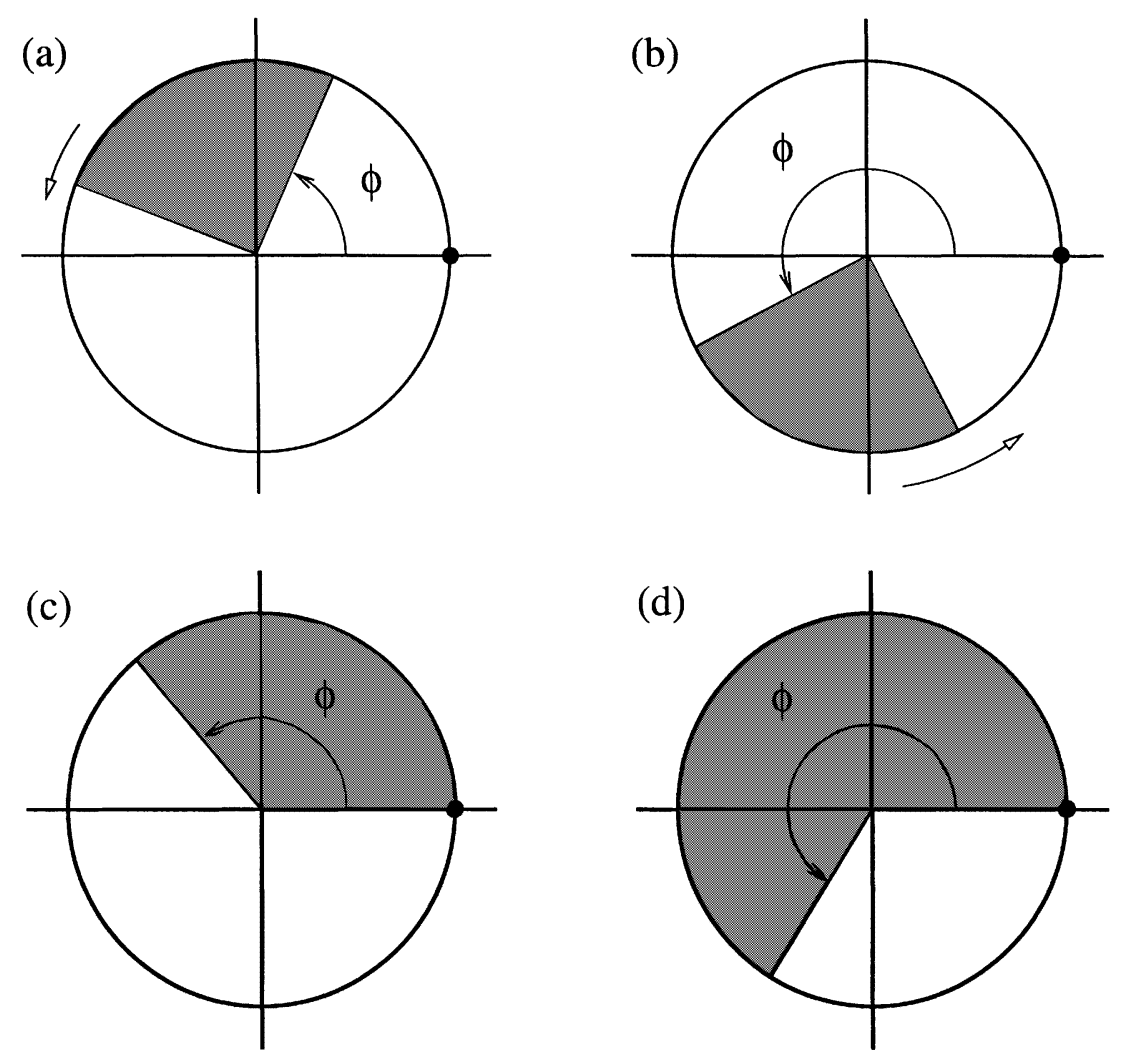

Fig. 1. The extent of the phonon band modes on the unit circle (see Eq. (3.3)). The angle $\phi$ corresponds to the bottom of the band on models with on-site potential (a, b), and to the top of the band on FPU models (c, d).

Fig. 1 shows the spreading of the linear modes in different models. For KG lattices with a soft nonlinear potential $V$, Fig. 1(a) corresponds to the case $\frac{2}{3}<\omega_{\mathrm{b}}<1$, and Fig. 1(b) to $\frac{1}{2}<\omega_{\mathrm{b}}<\frac{2}{3}$. The same figures are obtained for $\frac{2}{5}<\omega_{\mathrm{b}}<\frac{1}{2}$, and so on. For a hard nonlinear $V$, Fig. 1(a) is for $\omega_{\mathrm{b}}>2$ and Fig. 1(b) is for $1<\omega_{\mathrm{b}}<2$. For FPU lattices, where we drop the potential $V$ and $\lambda=1$, the linear band has a fixed width that spans from 0 to 2 $\left(\omega_{q}^{2}=4 \sin ^{2}(q / 2)\right)$. Fig. 1(c) corresponds then to FPU lattices with $\omega_{\mathrm{b}}>4$, and Fig. 1(d) to those with $2<\omega_{\mathrm{b}}<4$. For reasons that stem from Krein theory, it is useful to distinguish these regions of values of $\omega_{\mathrm{b}}$, and the results show different behavior in these regions.

We now show an overview of results in several models, but postpone all interpretation of the results, as well as further details on the analysis procedure itself, for a lengthier publication [20]. A preview of the level of the accuracy that can be achieved in this stability analysis of breathers was first shown in [19], for the case of a single-site breather in a simple harmonic plus cubic potential. Here we show the analogous analysis of single-site and two-site breathers in more complicated models.

\subsection{Lattices with soft on-site potentials}

When the potential $V$ is soft, the breather frequencies $\omega_{\mathrm{b}}$ lie below 1 . We have analyzed the stability of one-site breathers (that is, those emerging from a single oscillating site) for the two regions $\frac{2}{3}<\omega_{\mathrm{b}}<1$ and $\frac{1}{2}<\omega_{\mathrm{b}}<\frac{2}{3}$. The coupling has been varied from 0 to the limiting value imposed by the resonance condition 
Table 1

Spatial symmetry of the (localized) unstable mode for the one-site breather in different KG lattices with soft anharmonic potentials and linear coupling (for the case of $\phi^{4}$, only single-well orbits were considered)

\begin{tabular}{llll}
\hline Potential & Normalized form & $\frac{1}{2}<\omega_{\mathrm{b}}<\frac{2}{3}$ & $\omega_{\mathrm{b}}>\frac{2}{3}$ \\
\hline Cubic & $\frac{1}{2} u^{2}-\frac{1}{3} u^{3}$ & Symmetric, at -1 & - \\
Morse & $\frac{1}{2}\left(1-\mathrm{e}^{-u}\right)^{2}$ & Anti-symmetric, at +1 & Anti-symmetric, at +1 \\
Lennard-Jones & $\frac{1}{76}\left(\frac{1}{u^{12}}-\frac{2}{u^{6}}\right)$ & Anti-symmetric, at +1 & Anti-symmetric, at +1 \\
Cosine & $1-\cos (u)$ & - & - \\
$\phi^{4}$ & $\frac{1}{8} u^{4}-\frac{1}{4} u^{2}$ & Symmetric, at -1 & Anti-symmetric, at +1 \\
\hline
\end{tabular}

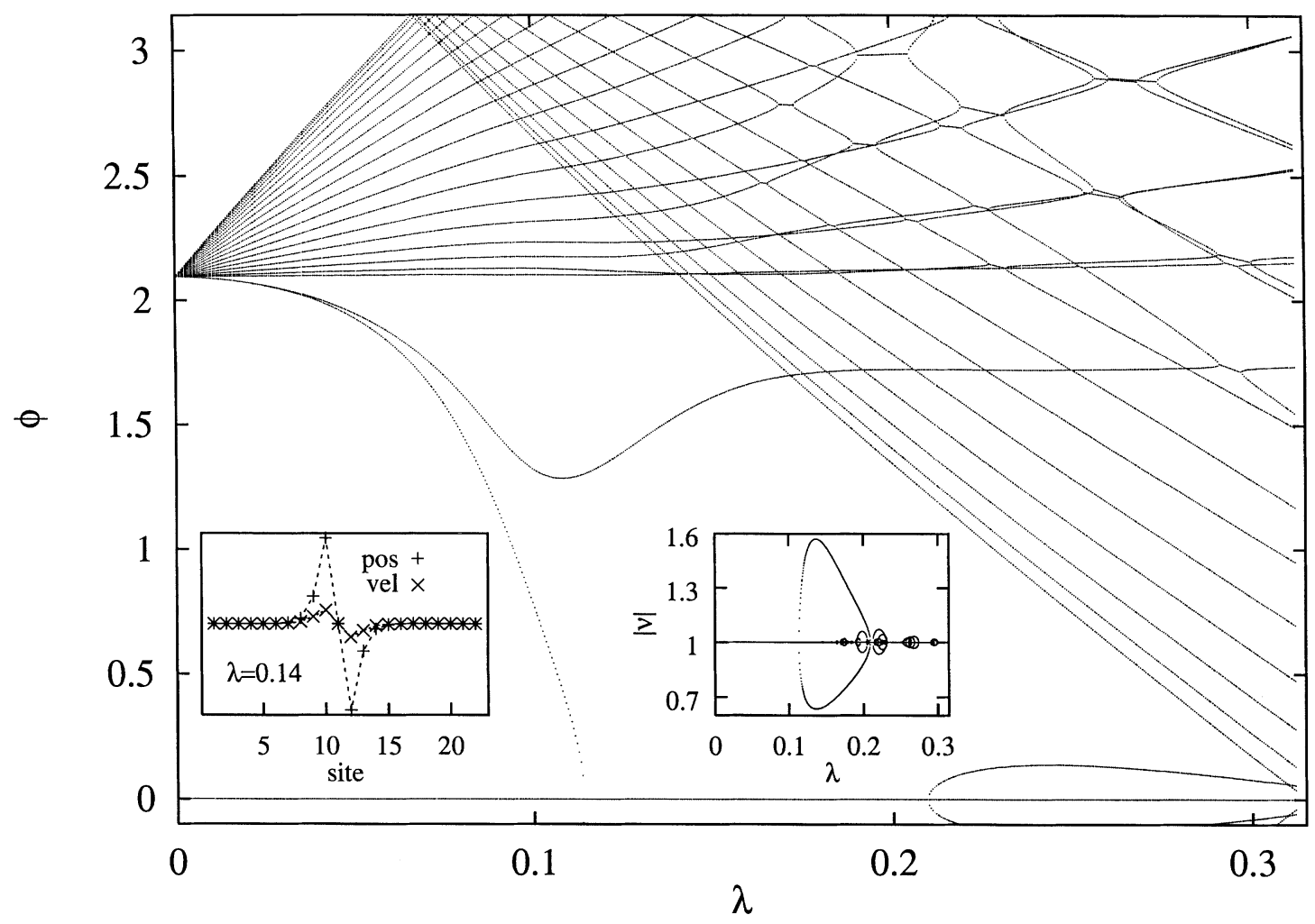

Fig. 2. The angle of the eigenvalues $v=\rho \mathrm{e}^{\mathrm{i} \phi}$ of the stability matrix $\mathcal{M}$, for the single-site breather of a Morse KG lattice with linear coupling potentials $\left(\omega_{\mathrm{b}}=0.75\right)$. One of the insets shows the absolute value of the eigenmodes. The other inset shows the spatially anti-symmetric localized mode that causes an instability at +1 . Note how in this case the stability is recovered at a larger coupling.

$$
2 \omega_{\mathrm{b}}=\sqrt{1+4 \lambda}
$$

where the breather solution can no longer be continued with our methods (see [11]). Note that this is the point at which the top of the phonon band reaches +1 on the unit circle (see Figs. 1(a) and 1(b)).

In the single-site breather the original couple of eigenvalues at +1 remains there and all possible localized instabilities will be caused by others, which necessarily will have to "detach" from the linear band. In all models, the eigenmodes coming from the bottom of the band are observed to be the ones which become progressively localized, as is expected from the fact that breathers in these potentials oscillate in-phase, and such is the symmetry 


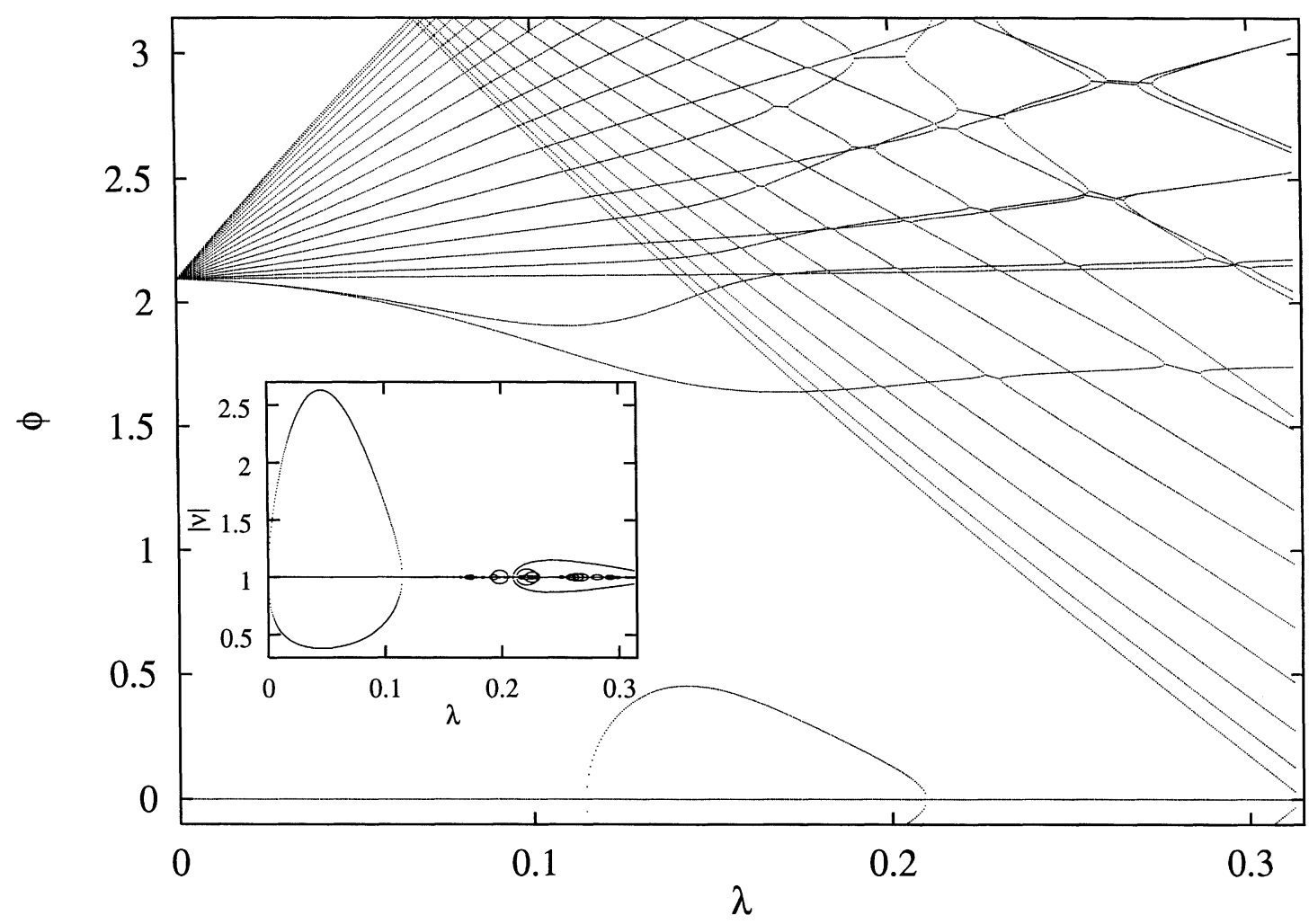

Fig. 3. Stability analysis of the two-site (in-phase) breather of a Morse KG lattice with linear coupling potentials $\left(\omega_{\mathrm{b}}=0.75\right)$. The breather is initially unstable, but then becomes stable when the two eigenmodes re-enter the unit circle. At larger coupling, it becomes unstable again.

of the lowest frequency linear modes of the lattice. But their behavior varies drastically from one model to another. In some models, one of the localized eigenmodes causes an instability when its associated eigenvalues collide at -1 (a period doubling bifurcation of the breather). In others, this collision occurs at +1 (a tangent bifurcation). Sometimes collisions are observed, but without any visible instability. Finally, we could also have no collisions at all, with the breather remaining stable along the full range of values of coupling. That is, up to the resonance condition given by Eq. (3.4).

Table 1 summarizes these findings in the models analyzed. It also details the spatial symmetry of the associated eigenvector that causes the instability: it can have a symmetric or anti-symmetric profile with respect to the breather center. Note that some models, like Morse and Lennard-Jones, may have two windows of stability, but we refer here to the first instability encountered.

In Figs. 2-4 we observe the evolution of the eigenvalues on the unit circle (the angles $\phi$ ), in different situations. We have calculated them on a relatively small lattice $(N=22)$ to have a better view of the discrete linear band, though this size is large enough not to distort the main results. We observe how the extended, "linear" modes behave following closely the linear dispersion relation (3.3). The ones departing from this behavior are observed to detach from the bottom of the band, and can be confirmed to be localized by looking at their associated eigenvector. The careful observer will also spot smaller instabilities in Figs. 2 and 3, caused by extended modes after their passing through -1 . These are just finite size effects, and vanish in the limit of an infinite lattice $(N \rightarrow \infty)$. This is properly justified in [20]. 


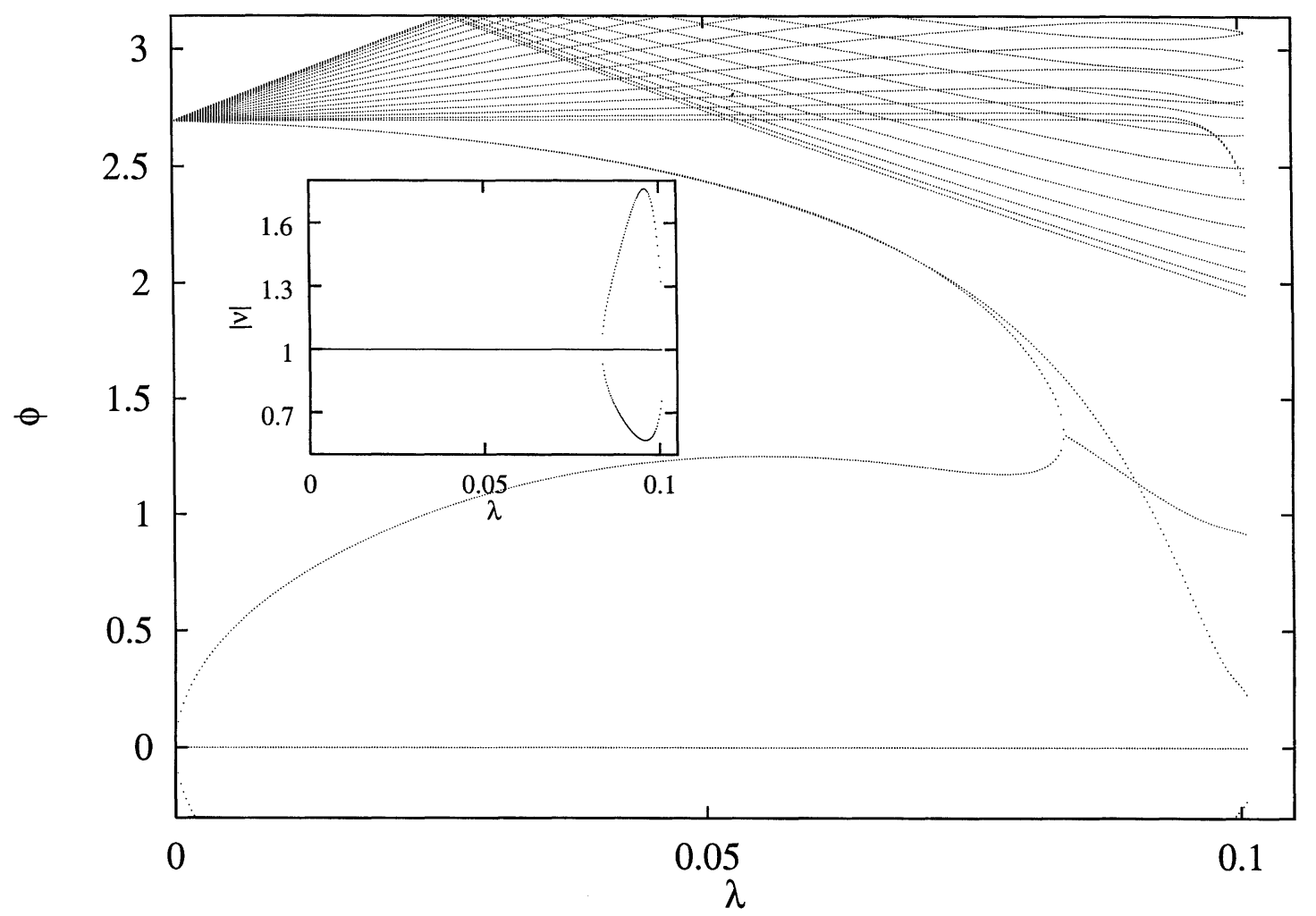

Fig. 4. Stability analysis of the two-site (out of phase) breather of a Morse KG lattice with linear coupling potentials $\left(\omega_{\mathrm{b}}=0.75\right)$. Here the breather is initially stable, and the first instability occurs when two eigenmodes collide at some angle on the unit circle (Hopf bifurcation). A later bifurcation at +1 prevents the continuation of the solution up to the coupling allowed by (3.4).

Fig. 3 shows the results for a two-site breather in which two neighboring sites are oscillating in-phase. Here we see that the two extra modes at +1 cause instability right from the beginning, though a stability window is later recovered at a higher value of $\lambda$, when these eigenvalues re-enter the unit circle. By contrast, the two-site breather in which the oscillators are in opposition of phase is initially stable (the extra eigenmodes at +1 start traveling along the unit circle) and becomes unstable at some higher coupling, as shown in Fig. 4. This behavior of two-site breathers near $\lambda=0$ can be understood analytically [20]. But a more interesting phenomenon was pointed out to us recently [21]. It is the fact that, on models exhibiting the tangent bifurcation of the single breather at +1 , the corresponding two-site (in-phase) breather also exhibits the same type of bifurcation at the same value of $\lambda$. For hard anharmonicity models, the same phenomenon occurs, but with the out-of-phase breather. This behavior will be extensively explained in a forthcoming paper [22].

\subsection{Lattices with hard on-site potentials}

The same analysis has been carried out for breathers in KG lattices with hard anharmonic potentials, in which the breather frequencies are above 1 . We report the results on the single-site breather in two models, both with a harmonic plus quartic on-site potential:

$$
V(u)=\frac{1}{2} u^{2}+\frac{1}{4} u^{4}
$$




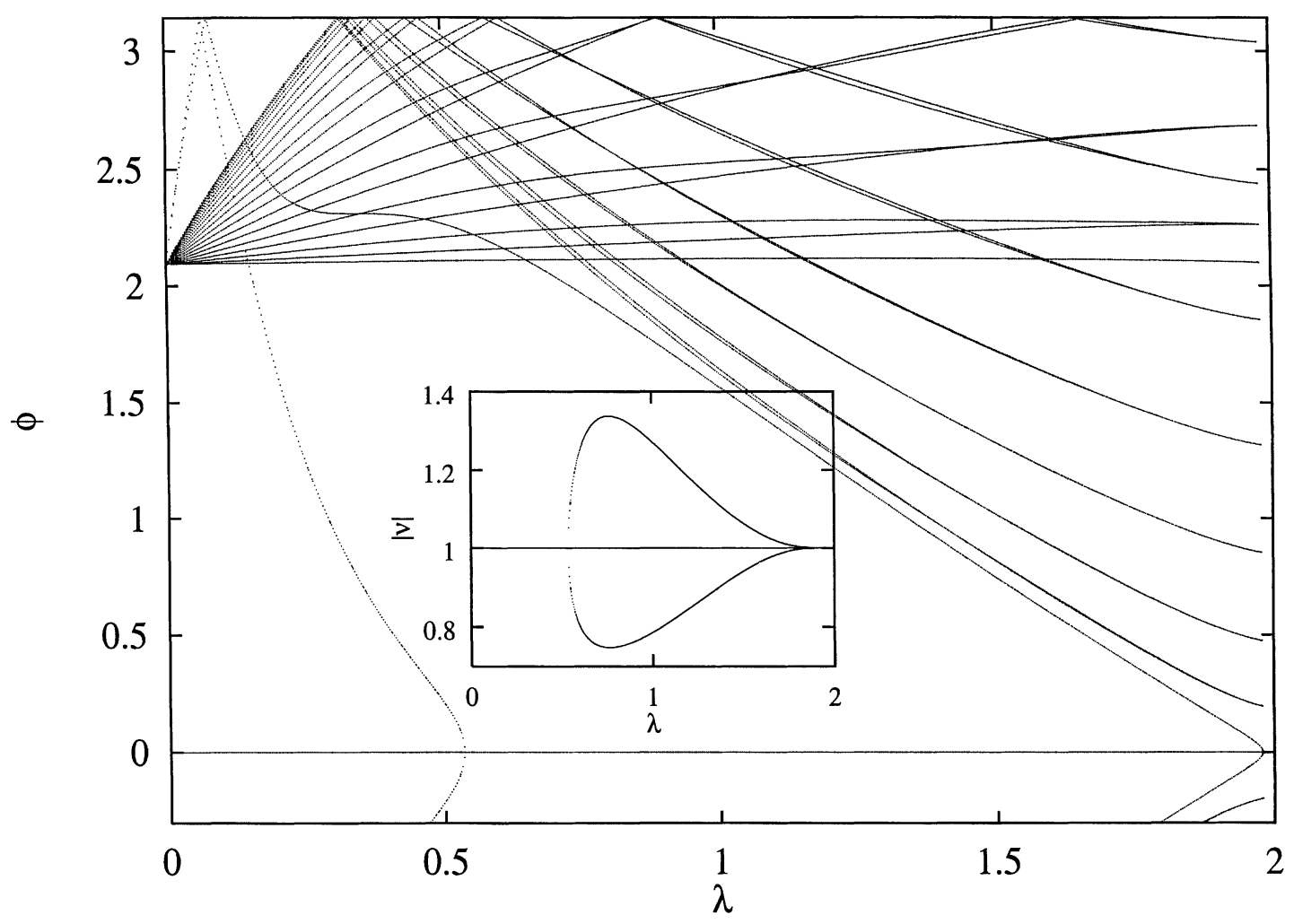

Fig. 5. Stability analysis of the one-site breather of a KG lattice with potentials given by (3.5) and (3.6).

but one of them with a linear coupling potential and the other with a hard nonlinear coupling of the same type as $V$ :

$$
W\left(u, u^{\prime}\right)=\frac{1}{2}\left(u-u^{\prime}\right)^{2}+\frac{1}{4}\left(u-u^{\prime}\right)^{4}
$$

It is interesting to introduce this latter model for several reasons. First, at large values of coupling, $\lambda \rightarrow \infty$, the model becomes equivalent to the FPU lattice with potential $W$ given by 3.6. Second, given the functional form of $V$ and $W$, at large values of the nonlinearity (or equivalently, when $\omega_{\mathrm{b}} \rightarrow \infty$ ) the system becomes equivalent to the simple "homogeneous" system given by the Hamiltonian

$$
H=\sum_{i} \frac{1}{2} \dot{u}_{i}^{2}+\frac{1}{4} u_{i}^{4}+\lambda \frac{1}{4}\left(u_{i}-u_{i+1}\right)^{4}
$$

Systems like this exhibit a separable property for periodic solutions, whereby breathers can be found by solving the dynamical part and the structural profile part separately [11,23]. These easily obtainable breathers serve as a double-check on the calculation of solutions of related models, like the one we consider here in Eq. (3.6).

The one-site breather in lattice (3.5) with linear couplings is observed to remain stable along the whole relevant range of values of $\lambda$, both in region $\omega_{\mathrm{b}}>2$ (Fig. 1(a)) as in region $1<\omega_{\mathrm{b}}<2$ (Fig. 1(b)). That is, no localized eigenmodes cause instability for values of coupling going from zero to the resonance condition

$$
\omega_{\mathrm{b}}=\sqrt{1+4 \lambda}
$$

which in hard anharmonicity KG lattices marks the limiting coupling beyond which the breather is not continuable with Newton methods (cf. (3.4) above). 
The situation changes when we consider the model (3.5) with the nonlinear coupling (3.6). Here, in region $1<\omega_{\mathrm{b}}<2$ (Fig. 1(b)) no instability is appreciated, but in region $\omega_{\mathrm{b}}>2$ (Fig. 1(a)) we observe a localized and spatially anti-symmetric eigenmode that collides at +1 and yields a tangent bifurcation of a very similar character to that encountered in the case of Morse, Lennard-Jones, and $\phi^{4}$ lattices. Again, the passage of these eigenmodes trough -1 does not yield instability, as would be expected in the general case. Fig. 5 shows the evolution of the eigenvalues for this case.

\section{Acknowledgements}

We would like to thank Thierry Cretegny for all the interesting and useful discussions on the stability problems. We acknowledge support from European Network ERBCHRXCT930331 and project No. PB95-0797 of the DGICYT (Spain). One of us (JLM) acknowledges a predoctoral grant from MEC, Spain.

\section{References}

[1] J.C. Eilbeck, P.S. Lomdahl, A.C. Scott, Physica D 16 (1985) 318.

[2] S. Takeno, K. Kisoda, A.J. Sievers, Prog. Theor. Phys. Suppl. 94 (1988) 242.

[3] A.J. Sievers, S. Takeno, Phys. Rev. Lett. 61 (1988) 970.

[4] S. Takeno, J. Phys. Soc. Jpn. 61 (1992) 2821.

[5] S. Takeno, K. Hori, J. Phys. Soc. Jpn. 60 (1991) 947.

[6] K. Hori, S. Takeno, J. Phys. Soc. Jpn. 61 (1992) 4263.

[7] R.S. MacKay, S. Aubry, Nonlinearity 7 (1994) 1623.

[8] S. Aubry, Physica D 71 (1994) 196.

[9] S. Aubry, Physica D 86 (1995) 284.

[10] S. Takeno, M. Peyrard, Physica D 92 (1996) 140.

[11] J.L. Marín, S. Aubry, Nonlinearity 9 (1996) 1501.

[12] L.M. Floría, J.L. Marín, P.J. Martínez, F. Falo, S. Aubry, Europhys. Lett. 36 (1996) 539.

[13] S. Flach, Phys. Rev. E 51 (1995) 3579.

[14] K.W. Sandusky, J.B. Page, K.E. Schmidt, Phys. Rev. B 46 (1992) 6161.

[15] O.A. Chubykalo, A.S. Kovalev, O.V. Usatenko, Phys. Lett. A 178 (1993) 129.

[16] F. Verhulst, Nonlinear Differential Equations and Dynamical Systems, 2nd ed., Springer, Berlin, 1996.

[17] V.I. Arnol'd, A. Avez, Ergodic Problems of Classical Mechanics, Benjamin, London, 1968.

[18] J. Moser, Comm. Pure Appl. Math. XI (1958) 81.

[19] J.L. Marín, S. Aubry, in: L. Vázquez, L. Streit, V.M. Pérez-García (Eds.), Nonlinear Klein-Gordon and Schrödinger Systems: Theory and Applications, World Scientific, Singapore, 1996, p. 317.

[20] J.L. Marín, T. Cretegny, S. Aubry, Linear stability analysis of discrete breathers, preprint, 1997.

[21] T. Cretegny, private communication.

[22] T. Cretegny, J.L. Marín, S. Aubry, Instability by a pinning mode and supermobility of a breather in a nonlinear lattice (1997), preprint, 1997.

[23] S. Flach, Phys. Rev. E 51 (1995) 1503.

[24] C. Baesens, S. Kim, R.S. MacKay, Localized modes on localized equilibria, Physica D 113 (1998) 242. 